Research Article

\title{
System Identification for Experimental Study for Polymerization Catalyst Reaction in Fluidized Bed
}

\author{
Ahmmed S. Ibrehem \\ Department of Chemical Engineering, UiTM University of Technologi Mara, 40450, \\ Shah Alam MALAYSIA
}

Received: 13rd May 2011; Revised: 27th July 2011; Accepted: 22th September 2011

\begin{abstract}
In this work, system identification method is used to capture the reactor characteristics of production rate of polyethylene (PE) based on published experimental data. The identification method is used to measure the percentage effect on the production rate of $\mathrm{PE}$ by measuring the effect of input factors of temperature of reaction, hydrogen concentration, and [Al]/[Ti] molar catalyst ratio. Temperature of reaction has big effects equal $52.4 \%$ on the output of the system and $47.6 \%$ on interaction of the system's parameters compare to other two factors. Also, hydrogen concentration has big effect equal $45.66 \%$ on the output of the system and $14.7 \%$ on interaction of the system's parameters. [Al]/[Ti] molar catalyst ratio has big effect on interaction of the system equal 28.6 and $1.94 \%$ on the output of the system but less than the reaction temperature and hydrogen concentration. All these results depend on experiment results and these results are very important in industrial plants. C2011 BCREC UNDIP. All rights reserved
\end{abstract}

Keywords: Catalysts, Co-catalyst, System identification, reaction temperature, hydrogen concentration

How to Cite: Ahmmed S. Ibrehem. (2011). System Identification for Experimental Study for Polymerization Catalyst Reaction in Fluidized Bed. Bulletin of Chemical Reaction Engineering \& Catalysis, $6(2): 137-146$

\section{Introduction}

Fluidized catalytic bed continuous reactors (FCR) entail very complex flow behavior (Ibrehem et al., 2008). Moreover, the presence of four phases in the reaction media complicates the transport phenomena involved which dictates the need for a thorough understanding of parameters effect on production rate and crystallization characteristics.

System identification method (Ibrahem \& Hikamt, 2009) should have sufficient accuracy of prediction, wide applicability, the minimum assumption about the most parameters effects like temperature of reaction, hydrogen concentration, and [Al]:[Ti] molar ratio. In the area of production rate for polyethylene system and identify which is most input variable effects on the production rate by using experiment work was very little. In 2009 , Zohuri group made a very good experiment work design techniques for polymerization process based on experimental data only. We depend on this important work to specify which is the most active input parameters whose effect on the system depends on experimental results and new identification method to specify the percentage effect of production of high molecular weight of

\footnotetext{
* Corresponding Author.

E-mail address: ahmadsaadi1@yahoo.com (A.S. Ibrehem)
} 
polyethylene.

High molecular weight polymers and their morphological development are the most important achievements of heterogeneous catalyst polymerization of the Ziegler-Natta type (Peacock, 2000; Jamjah et al., 2008; Dasilva \& Defigueiredo, 2002; Shiraishi et al.,1990). $\mathrm{MgCl}_{2}$ and $\mathrm{SiO}_{2}$ are the most common bisupports used to heterogenize olefin polymerization catalysts (Pater et al., 2003; Jamjah et al., 2006; Zohuri et al., 2006; Moore, 2005), and $\mathrm{SiO}_{2}$ is also used to support metallocene and late transition metal catalysts (Galli \& Vecellio, 2004; Barbe et al., 1986). The morphological development of the catalyst originally obtained from its support could replicate the polymer in controlled conditions. Heterogeneous catalyst particles break up during the early stages of the polymerization, possibly due to the chemical reaction of the catalyst component, mechanical stress and also polymer growth, respectively [Silva et al., 2005; Shaotian et al., 2001). In Ziegler-Natta polymerization, the rate/time profiles are mainly a decay type with very high polymerization activity at the early stage of the reaction. Therefore, the fast growth of the polymer into the pore and channel of the catalyst could fragmentize the catalyst particles. To achieve a reasonable morphology, the fragmentation must be controlled, since uncontrolled fragmentation may produce fine particles. Using $\mathrm{SiO}_{2}$ and $\mathrm{MgCl}_{2}$ components of a solid, support not only raised the activity of the catalyst but also improved its morphology. $\mathrm{SiO}_{2}$ also improved the mechanical and thermal stability of the catalyst (Kono et al., 2001; Yamahiro et al., 1999). Interaction between co catalyst (TEA) and catalyst are very important and explained very well in Ibrahem et al. (2009) and Hatzantonis et al. (2000).

Morphological development of support, catalyst and polymer is the major area of study nowadays. Ultra high molecular weight polyethylene has many desirable physical and mechanical characteristics comparable to some engineering plastics (Fukuda et al., 2003; Zohuri et al., 2003; Zohuri et al., 2001). The present work studies the effect of co catalyst $\mathrm{Al} / \mathrm{Ti}$ flow rate, temperature input and hydrogen concentration on production rate of polyethylene.

\section{Dynamic analysis and identification for mathematical model}

System identification involves building a dynamical model from an input/output data and without use of any laws concerning the fundamental nature and properties of the nonlinear system. The batch reactor process involves many variables, which contribute to its operation, and this makes it a Single-Input SingleOutput (SISO) process. These variables are classified as follows: Input or manipulated variables (MVs) are chosen from those variables that have direct effect on the process performance, and practically easy to actuate. In our case, these are: percent ratio of $\mathrm{Al} / \mathrm{Ti}$ as a co catalyst $(\mathrm{Qc})$, temperature input and concentration of hydrogen. The controlled variables (CVs) are selected from the process outlet streams that are usually of foremost importance such as the production polyethylene (PE). A schematic diagram representing the previously mentioned variables is shown in Figure (1).New knowledge in this work shows effects of three important inputs variables of polymerization system on the rate of production compared to other works.

So, the identification steps can be defined for PASA as follows (Ibrahem \& Hikamt, 2009):

1. To make actual calculations for the system from nominal conditions xn,i to calculate yn,i.

2. To repeat step one with $+\mathrm{H} \%$ perturbed parameter input vector $(\mathrm{H} \times \mathrm{xn}, \mathrm{i})$ to produce the $+\mathrm{H} \%$ perturbed output yi matrix.

3. To subtract each element from matrix in first step from the corresponding element in matrix in second step and divide the difference by yn,i. (yi- yn,i) / yn,i.

4. Similarly calculate the change in parameters (xi- xn,i) / xn,i.

5. Divide each element in third step by fourth step to produce the sensitivity matrix $\mathrm{k}$ that depends completely on scale matrix without using any a proper factor.

6. Calculate $\mathrm{k}$ matrix for negative direction which mean calculate $\mathrm{k}+\mathrm{H} \%$ and $\mathrm{k}-\mathrm{H} \%$ so as, we can check the effect of each parameters on output as seen in Table (2) .

7. Then find kaverage slope to find average angle $(\theta)$ that is represented overall effect of each parameters on the measured output $(\theta 1$ represents the effects of $\mathrm{Al} / \mathrm{Ti}, \theta 2$ represents the effects of reaction temperature and $\theta 3$ represents the effects of hydrogen concentration on the system as shown in Figure 9 . From parameters average slope analysis, we can see a preliminary partitioning of estimating into different groups that depend on slope angle $(\theta)$ of kaverage and these groups can be specified as follows:

$(\theta) \geq 20^{\circ}$ : Large effects on the system. $20^{\circ}>(\theta)>15^{\circ}$ : Middle effects on the system. 


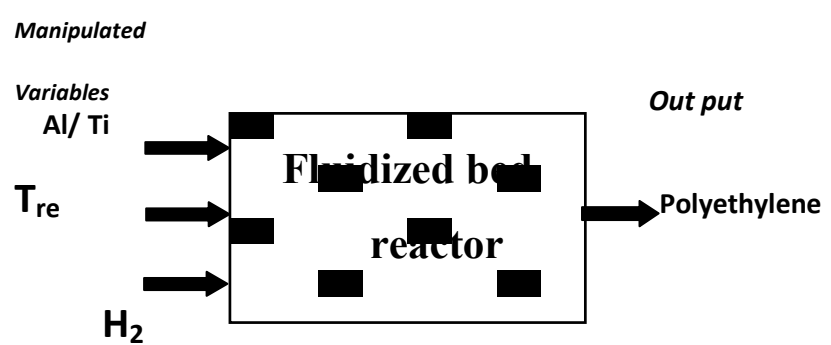

Figure 1. Representation of variables in polymerization system as a MISO system

$15^{\circ} \geq(\theta) \geq 10^{\circ}$ : Weak effects on the system.

$10^{\circ}<(\theta)$ : Cannot be established.

$\theta$ total $=\theta$ in the system $+\theta$ interaction

$\theta$ total $=\theta 1+\theta 2+\theta 3$

\section{Reaction Kinetics}

In the present study, a comprehensive mechanism is considered to describe the copolymerization kinetics of ethylene and 1-butane over a Ziegler-Natta catalyst with two different catalyst porosity and rigid catalyst sites based on the kinetic model proposed by Mc-Auley et al. (1990). Rates of formation, initiation, propagation and chain transfer are different for each site type. This mechanism comprises of series and parallel elementary reactions as listed in Table 1 . The rate constants used in this study are those given by Kinetic and Pseudokinetic (Mc-Auley et al., 1990) and the activation energies are taken from for porous catalysts the effects of adsorption, desorption and surface reaction are included in the rate of chemical reaction. The reactions are listed in Table 1. From the kinetic mechanism, the rate expression for the active sites $\left(r_{a}\right)$ for each species can be written as follows:

$r_{a}=\begin{gathered}\text { Active site formation } \\ \text { consumption }\end{gathered}$

$$
k^{n}\left[P^{0}\right]-\sum_{j=1}^{2} k_{j}^{i}\left[P_{0}\right]\left[M_{j}\right]
$$

The derivation of the rate expressions based on the kinetic mechanism for rigid catalyst is given in Table 2. The over all rate expression for live and dead moment is represented in equation (3) and (4) respectively, as seen below. Rate expressions for live moments is given as:

$$
\begin{aligned}
R_{\mu_{n, i}}^{k}= & -r_{P_{0}} \times P_{n, i}-\left(r_{H_{2}} \times P_{n, i}^{k}\right)-\left(r_{P_{n, i}} \times P_{n, i}\right)+\delta(n)\left(\sum_{j=1}^{n} k^{h} \mu_{0} M_{i}+k^{i} M_{i}\right) \\
& (1-\delta(n)) \sum_{j=1}^{n} k_{j}^{P} M_{i} P_{n-1, j}-\sum_{j=1}^{n} k_{j}^{P} M_{j} P_{n, j}
\end{aligned}
$$

where $\delta(n)$ is the Kronecker's delta function (e.g., $\delta$ $(n)=1$ for $\mathrm{n}=1$ and $\delta(n)=0$ for $\mathrm{n} \neq 1)$. Rate expressions for dead moments is given as:

$$
R_{v_{n, i}}^{k}=\left(k^{f}[M]+k^{h}\left[H_{2}\right]\right) \mu_{n}
$$

The dynamic mass balance for the catalyst is given as:

$$
\frac{d C_{c a t}}{d t}=\frac{F_{c a t}}{W_{s}}-\frac{Q_{0} C_{c a t} \rho_{c a t}}{W_{s}}
$$

Similarly, the mass balance for the potential active sites and active sites are:

$$
\frac{d\left[P^{0}\right]}{d t}=\frac{F_{c a t} P_{i n}^{0}}{W_{S}}-\frac{Q_{0} P^{0} \rho_{c a t}}{W_{S}}-r_{P^{0}}
$$

and

$$
\frac{d\left[P_{0}\right]}{d t}=\frac{F_{c a t} P_{0}}{W_{S}}-\frac{Q_{0} P_{0} \rho_{c a t}}{W_{S}}-r_{P_{0}}
$$

The rate expression for each species of porous catalyst can be written and the rate expression for the active sites $r_{a}$ can be written as follows:

$M_{j}=\left[\frac{m_{T}-P_{n, i} / K_{D}}{1+P_{A} K_{A}}\right] P_{A} K_{A}$

From the kinetic mechanism, the rate expression for the active sites $r_{a}$ can be written as follows:

$r_{a}=$ Active site formation - Active site consumption

$$
\begin{aligned}
& =\quad k^{n} P^{0}-\sum_{j=1}^{2} k_{j}^{i} P_{0}\left[\frac{M_{T}-P_{n, i} / k_{D}}{1+P_{A} k_{A}}\right] P_{A} k_{A} \\
& =\quad k^{n} P^{0}-\sum_{j=1}^{2} k_{j}^{i} P_{0} M_{j}
\end{aligned}
$$




\section{Experimental study}

$\mathrm{SiO}_{2}$ (PQ3050) was acquired from $\mathrm{PQ}$ Corporation (USA). The compound was calcinated at about $400{ }^{\circ} \mathrm{C}$ for nearly $5 \mathrm{~h}$ before use [19]. Spherical adducted $\mathrm{MgCl}_{2} \cdot \mathrm{nC}_{2} \mathrm{H}_{5} \mathrm{OH}$ was prepared according to literature (Shiraishi et al., 1990). Slurry polymerization of ethylene was carried out in a 1-L stainless-steel reactor of Buchi (BDS300), a semibatch type, equipped with a stirrer speed control, temperature and pressure control. The reactor was purged with nitrogen. A volume of 400 $\mathrm{mL}$ heptane was charged into the reactor and degassed at least three times. When the polymerization temperature reached the required temperature, the catalyst components were added in the following order; TEA, the solid catalyst, and hydrogen (if any). All the reagents were added as slurry in n-heptane or a gas. The pressure inside the reactor was maintained constant with the monomer gas. Therefore, the required amount of the monomer to feed into the reactor is equal to the consumption of the monomer. At the end of the reaction time, mainly one hour, the monomer feed was stopped. The polymerization waste ruminated by draining the slurry polymer into a small volume of acidified methanol. The polymer obtained was filtered and dried at $70^{\circ} \mathrm{C}$ for overnight (Zohuri et al., 2001).

\section{Catalyst preparation}

The $\mathrm{SiO}_{2} / \mathrm{MgCl}_{2} / \mathrm{TiCl}_{4}$ catalyst was prepared using the adducted spherical $\mathrm{MgCl}_{2} \cdot \mathrm{nC}_{2} \mathrm{H}_{5} \mathrm{OH}$. The ethanol was chemically removed during the catalyst preparation procedure. The calcinated $\mathrm{SiO}_{2}(2.5 \mathrm{~g})$ and $\mathrm{MgCl}_{2} \cdot \mathrm{nC}_{2} \mathrm{H}_{5} \mathrm{OH}$ (2.5 g) were added to a catalyst preparation reactor containing a sinter glass heated with a jacket. The chemicals were suspended in toluene $(100 \mathrm{~mL})$, and then $\mathrm{TiCl}_{4}(40 \mathrm{~mL})$ was added dropwise, while, the contents of the reactor were stirred at $5{ }^{\circ} \mathrm{C}$. The temperature was raised to $115{ }^{\circ} \mathrm{C}$ in increments of $20{ }^{\circ} \mathrm{C}$, while stirring at each step for at least one hour. The product was filtered, and washed with nheptane $(100 \mathrm{~mL})$. Toluene $(100 \mathrm{~mL})$ and $\mathrm{TiCl}_{4}(40$ $\mathrm{mL}$ ) were added at $40{ }^{\circ} \mathrm{C}$. The temperature was raised to $115^{\circ} \mathrm{C}$ incrementally, as before. The final catalyst was filtered, washed with n-heptane to completely remove unreacted $\mathrm{TiCl}_{4}$, and dried. All the steps were carried out under an atmosphere of dried $\mathrm{N}_{2}$ (Zohuri et al., 2001). Co catalyst is very important to be added with catalyst as particular ratio $2 \mathrm{~g}$ of catalyst per $0.8-1 \mathrm{gm}$ of co catalyst as can be shown in the Figure 2 .

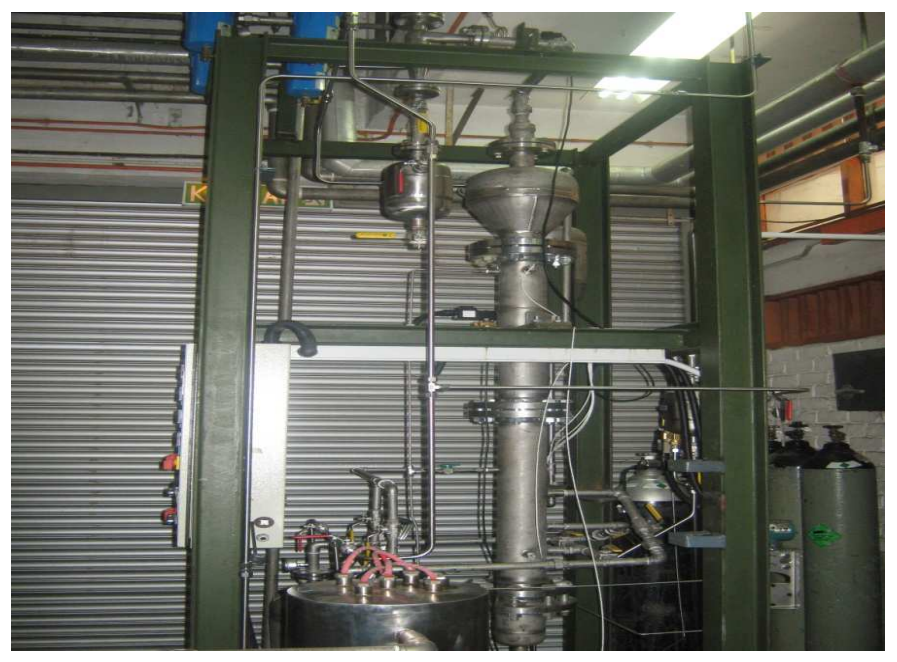

Figure 2. Side view for experimental study

\section{Results and discussion}

Figure 3 shows the relation between $\mathrm{Al} / \mathrm{Ti}$ and production rate of $\mathrm{PE}$. The optimum value for $\mathrm{Al} / \mathrm{Ti}$ $=770 / 1$ molar ratio gives production rate of $\mathrm{PE}=$ 1845 at constant reaction temperature $=60{ }^{\circ} \mathrm{C}$. Depend on paragraph 2, can see all calculations in Table 3. The average slope $=0.52$ and $\theta$ average $=$ $27.49^{\circ}$, that is, mean $\mathrm{Al} / \mathrm{Ti}$ ratio has large effects on the system $=30.54 \%$. Figure 4 shows the relation between hydrogen concentration and production rate of $\mathrm{PE}$ which is an inverse relation; when the concentration of hydrogen increase, the production rate will be decreased because addition of $\mathrm{H} 2$ closes the rate of live reaction and make dead polymer to stop polymerization by competing with monomer in adsorption on active center that reduce polymerization. Also, in Table 4 average slope $=1.3929$ and $\theta$ average $=54.32^{\circ}$, that is, mean $\mathrm{H}_{2}$ concentration has large effects on the system = $60.36 \%$. Figure 5 shows the relation between reaction temperature and production rate of $\mathrm{PE}$. The optimum value for reaction temperature $=60$ ${ }^{\circ} \mathrm{C}$. gives production rate of $\mathrm{PE}=1845$ at $\mathrm{Al} / \mathrm{Ti}$ $=770 / 1$ molar ratio. In Table 5 the average slope $=$ 1.4885 and $\theta$ average $=89.99^{\circ}$, that is, mean reaction temperature has largest effects on the system $=99.9 \%$. Figure 6 represents all three effects inside the system that are represented by average angle slope. From equation (1):

\footnotetext{
$\theta$ total $=89.99^{\circ}+54.32^{\circ}+27.49^{\circ}=171.8^{\circ}$

$\theta$ in the system $=90^{\circ}$

- interaction $=\theta$ total $-\theta$ in the system

$\theta$ interaction $=171.8^{\circ}-90^{\circ}=81.8^{\circ}$

$\theta$ interaction / $\theta$ total $\approx \theta 2 / \theta 1$
} 
Table 6 shows the percentage effects of a temperature, hydrogen concentration and catalyst ratio in the system and interaction between these inputs variables. $\mathrm{Al} / \mathrm{Ti}$ has a big interaction effect $28.6 \%$ on the temperature and hydrogen concentration because co catalyst enters as a protect catalyst from poison that leads to increase temperature of reaction that effects on rate of site activation reactions as can be explained in paragraph 2.

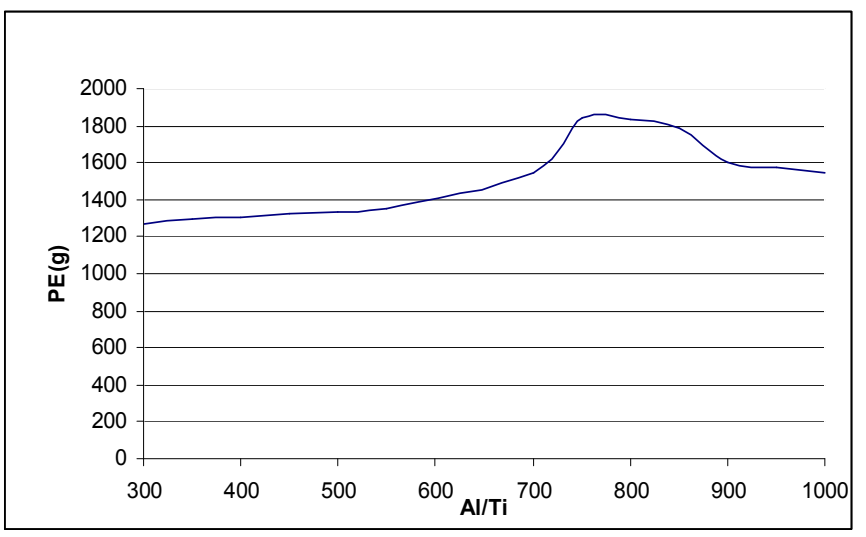

Figure 3. Effect of Al/Ti molar ratio on production rate of polyethylene, temperature $60^{\circ} \mathrm{C}$

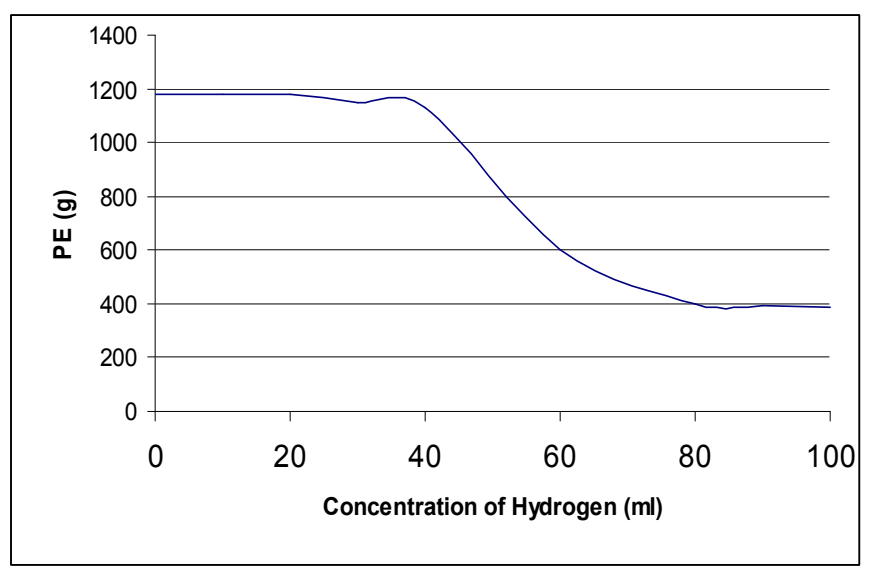

Figure 4. Effect of $\mathrm{H}_{2}$ concentration on production rate of polyethylene, temperature $60^{\circ} \mathrm{C}, \mathrm{Al} / \mathrm{Ti}=$ $770: 1$
Table 1. Numerical values of the kinetic rate constants for adsorption, surface and desorption reactions inside the catalyst layers

\begin{tabular}{ccc}
\hline Rate constant $\left(\mathrm{s}^{-1}\right)$ & \multicolumn{2}{c}{ Site type } \\
\cline { 2 - 3 } $\begin{array}{c}\text { Activation energy }(\mathrm{E}) \\
\text { (kcal/mol) }\end{array}$ & 1 & 2 \\
E & & 0.001 \\
Reactant-adsorption $\left(K_{A}\right)$ & 9 & 9 \\
Surface reaction $\left(K_{s}\right)$ & 0.001 & 0.001 \\
$\mathrm{E}$ & 9 & 9 \\
Product desorption $\left(K_{D}\right)$ & 0.00047 & 0.00047 \\
$\mathrm{E}$ & 9 & 9 \\
$M_{j}+s \rightleftarrows M_{j, s}$ & Reactant & \\
$M_{j, s} \longrightarrow P_{n, i, s}$ & adsorption \\
$P_{n, i, s}$ & surface re- \\
& action \\
\hline$P_{n, i}+S$ & product de- \\
& sorption \\
\hline
\end{tabular}

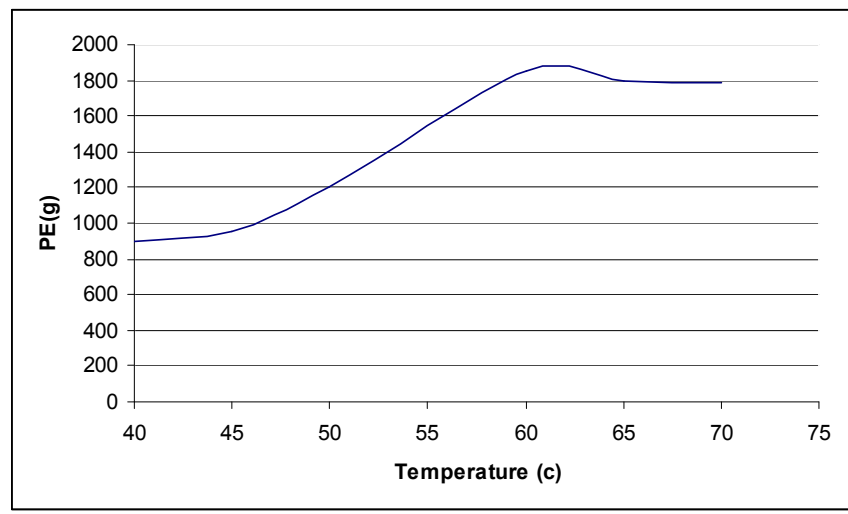

Figure 5. Effect of temperature on production rate of polyethylene, $\mathrm{Al} / \mathrm{Ti}=770: 1$

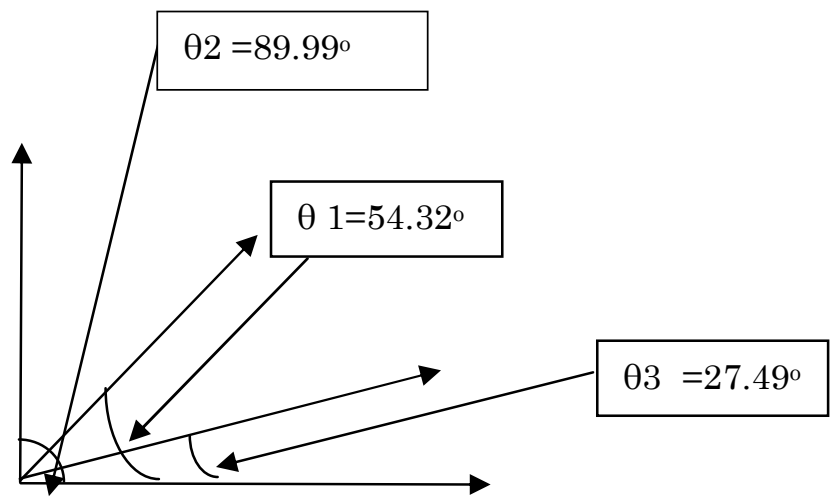

Figure 6. Representation of the average angle slope 
Table 2. Kinetic mechanism of olefin copolymerization for rigid Ziegler-Natta catalyst

Spontaneous activation:

$$
P^{0} \stackrel{k^{n}}{\longrightarrow} P_{0}
$$

Initiation: $P_{0}+M_{j} \stackrel{k_{j}^{i}}{\longrightarrow} P_{1, j,} \quad \mathrm{j}=1,2$

(Formation of $P_{1, i}: P_{0}+M_{i} \stackrel{k_{i}^{i}}{\longrightarrow} P_{1, i,} \quad$ i=1,2)

Propagation:

$$
P_{n, i}+M_{j} \stackrel{k_{i, j}^{P}}{\longrightarrow} P_{n+1, j,}, \quad i, j=1,2, n=1,2, \ldots, \infty
$$

(Consumption of

$$
\left.P_{1, i}: P_{1, i}+M_{i} \stackrel{k_{i, j}^{P}}{\longrightarrow} P_{2, j,} \quad i, j=1,2, n=1,2, \ldots, \infty\right)
$$

(Formation of

$$
\left.P_{n, i}: P_{n-1, i^{\prime}}+M_{i} \stackrel{k_{i, i}^{P}}{\longrightarrow} P_{n, i,} \quad i^{\prime}, i=1,2, n=1,2, \ldots, \infty\right)
$$

Chain transfer:

$$
P_{n, i}+M_{j} \stackrel{k_{i, j}^{f}}{\longrightarrow} P_{1, j}+Q_{n,} \quad i, j=1,2, n=1,2, \ldots, \infty
$$

$$
P_{n, i}+H_{2} \stackrel{k_{i}^{h}}{\longrightarrow} P_{0}^{*}+Q_{n,} \quad i, j=1,2, n=1,2, \ldots, \infty
$$

(Formation of

\begin{tabular}{|c|c|c|c|}
\hline Ratio AlTi (xi) & $(x i-x n, i) / x n, i$ & PE-mmol(yi- yn,i) / yn,i & Slope (k) \\
\hline 300 & 0.538 & 0.117 & 0.217 \\
\hline 350 & 0.461 & 0.096 & 0.208 \\
\hline 400 & 0.384 & 0.088 & 0.229 \\
\hline 450 & 0.307 & 0.074 & 0.241 \\
\hline 500 & 0.23 & 0.071 & 0.308 \\
\hline 550 & 0.153 & 0.065 & 0.424 \\
\hline 700 & 0.076 & 0.053 & 0.697 \\
\hline 750 & 0.153 & 0.292 & 1.908 \\
\hline 800 & 0.23 & 0.289 & 1.256 \\
\hline 850 & 0.307 & 0.205 & 0.667 \\
\hline 900 & 0.384 & 0.107 & 0.278 \\
\hline 950 & 0.461 & 0.086 & 0.186 \\
\hline 1000 & 0.538 & 0.079 & 0.146 \\
\hline Kaverage & & & 0.52 \\
\hline$\theta$ Average & & & $27.49 \mathrm{o}$ \\
\hline $\begin{array}{c}\% \text { effect }(\theta \text { Aver- } \\
\text { age } \times 100) / 90^{\circ}\end{array}$ & & & 30.54 \\
\hline
\end{tabular}

$$
P_{1, i}: P_{n, i^{\prime}}+M_{i} \stackrel{k_{i, i}^{f}}{\longrightarrow} P_{1, i}+Q_{n,} \quad i^{\prime}, j=1,2, n=1,2, \ldots, \infty \text { ) }
$$

(Consumption of $P_{1, i}: P_{1, i}+M_{j} \stackrel{k_{i, i}^{f}}{\longrightarrow} P_{1, j}+Q_{1,} \quad i, j=1,2$ )

(Consumption of

$$
P_{1, i}: P_{1, i}+H_{2} \stackrel{k_{i}^{h}}{\longrightarrow} P_{0}^{*}+Q_{1,} \quad i, j=1,2
$$

Table 3. Calculation of the percent difference for catalyst ratio against output profile 
Table 4. Calculation the percent difference for hydrogen concentration against output profile

\begin{tabular}{|c|c|c|c|}
\hline Hydrogen concentration $(\mathrm{ml})$ & $(\mathbf{x i}-\mathbf{x n}, \mathbf{i}) / \mathrm{xn}, \mathbf{i}$ & PE-mmol(yi- yn,i) / yn,i & Slope (k) \\
\hline 0 & 1 & 1.275 & 1.275 \\
\hline 10 & 0.8 & 1.225 & 1.531 \\
\hline 20 & 0.6 & 1.125 & 1.875 \\
\hline 30 & 0.4 & 0.9 & 2.25 \\
\hline 40 & 0.2 & 0.5 & 2.5 \\
\hline 60 & 0.2 & 0.3 & 1.5 \\
\hline 70 & 0.4 & 0.4 & 1 \\
\hline 80 & 0.6 & 0.5 & 0.833 \\
\hline 90 & 0.8 & 0.512 & 0.64 \\
\hline 100 & 1 & 0.525 & 0.525 \\
\hline Kaverage & & & 1.3929 \\
\hline$\theta$ Average & & & $54.32 \mathrm{o}$ \\
\hline$\% \operatorname{effect}(\theta$ Average $\times 100) / 900$ & & & $60.36 \%$ \\
\hline
\end{tabular}

Table 5. Calculation of the percent difference for reaction temperature against output profile

\begin{tabular}{cccc}
\hline Temperature (co) & $\mathbf{( x i}-\mathbf{x n}, \mathbf{i}) / \mathbf{x n}, \mathbf{i}$ & PE-mmol(yi- $\mathbf{y n}, \mathbf{i}) / \mathbf{y n}, \mathbf{i}$ & Slope $(\mathbf{k})$ \\
\hline 40 & 0.272 & 0.512 & 1.882 \\
45 & 0.181 & 0.489 & 2.701 \\
50 & 0.09 & 0.368 & 4.088 \\
60 & 0.09 & 0.022 & 0.244 \\
65 & 0.181 & 0.001 & 0.005 \\
70 & 0.272 & 0.003 & 0.011 \\
Kaverage & & & 1.4885 \\
$\theta$ Average & & & $89.99 \mathrm{o}$ \\
\hline \% effect( $\boldsymbol{\theta}$ Average $\times \mathbf{1 0 0}) / \mathbf{9 0 0}$ & & & $99.9 \%$ \\
\hline
\end{tabular}

Table 6. Calculation of the percent effects for in the system and interaction between the inputs variables

\begin{tabular}{cccc}
\hline Action & Temperature & Hydrogen concentration & Ratio AL/Ti \\
\hline $\begin{array}{c}\text { Percentage effects } \\
\text { inside the system } \\
\begin{array}{c}\text { Percentage } \\
\text { interaction effects }\end{array}\end{array}$ & 52.4 & 45.66 & 1.94 \\
\hline
\end{tabular}

very flexible analysis of polymerization system to reach to important results that can be used and which depend in industry. The most active input parameter is reaction temperature compare to other factors because it is effected $52.4 \%$ in the system for production $\mathrm{PE}$ and $47.6 \%$ for interaction effects between the two factors. Also, hydrogen input factor has big effect on the system because it is effected $45.66 \%$ directly in the rate of live and dead polymer for production $\mathrm{PE}$ and $14.7 \%$ for interaction effects between the two factors. $\mathrm{Al} / \mathrm{Ti}$ ratio has small effect $1.94 \%$ in the system but it is big effect $28.6 \%$ in interaction sides between the two factors (reaction temperature and hydrogen concentration). Reaction temperature and hydrogen concentration mainly effect for all active sites of catalyst surface reaction. All these results are very important in industrials of polymerization system. The initial stage of the polymerization, including the reaction of the catalyst with the cocatalyst and the high polymerization activity, broke up the catalyst particles into smaller particles of irregular shape and size. The morphology of the polymer particles was almost the same as that of the catalyst, which reacted 
with the cocatalyst at the early stage of the polymerization. In addition, the morphology of the particles may be affected by polymerization conditions, such as temperature and monomer pressure. However, the parameters of the initial stages may also be critical.

\section{Nomenclatures}

$k_{i} \quad$ Rate constant of reaction, $1 / \mathrm{s}$

$k_{A} \quad$ Thermal conductivity between layers of

$k_{A} \quad$ catalyst particles, $\mathrm{J} / \mathrm{m}^{2} / \mathrm{s} / \mathrm{k}$

$\mathrm{k}^{\mathrm{n}} \quad$ Rate constant of spontaneous reaction $1 / \mathrm{s}$

$\mathrm{k}^{\mathrm{f}} \quad$ Rate constant of chain transfer $1 / \mathrm{s}$

$\mathrm{K}^{\mathrm{h}} \quad$ Rate constant of chain transfer to hydrogen $1 / \mathrm{s}$

$\left[M_{e}\right]$ Active metal concentration, $\mathrm{mol} \mathrm{Me} / \mathrm{m}^{3}$

$\left[M_{i}\right]$ "I"monomer concentration, $\mathrm{mol} \mathrm{Me} / \mathrm{m}^{3}$

$P_{A} \quad$ Partial pressure of $\mathrm{A}$ in gas phase

Po Potential active sites $\mathrm{kmol} / \mathrm{m}^{3}$

$\mathrm{P}_{\mathrm{o}} \quad$ Active sites concentration $\mathrm{kmol} / \mathrm{m}^{3}$

$S_{P}^{K} \quad$ Concentration of potential " $\mathrm{k}$ " catalyst

$S_{P} \quad$ active sites, $\mathrm{mol} / \mathrm{m}^{3}$

$r_{a} \quad$ Rate expression for the active sites kmol/kg.catalyst

$\mathrm{R}^{\mathrm{k}_{\mu \mathrm{n}, \mathrm{i}} \quad \text { Rate expression for live moments }}$

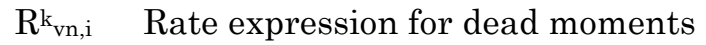

$R_{X}^{K} \quad$ Reaction rate of species $\mathrm{X}$ at " $\mathrm{k}$ " catalyst activesites, $\mathrm{mol} / \mathrm{m}^{3} / \mathrm{s}$

\section{References}

[1] Ibrahem, A.S. \& Hikamt, S.I. (2009). New Dynamic Analysis and System Identification Of Biodiesel Production Process From Palm Oil. Bulletin Chemical Reaction Engineering and Catalysis, 4(2): 61-68

[2] Ibrehem, A.S., Hussain, M.A., \& Ghasem, N.M. (2008). Mathematical model and advanced control for gas-phase olefin polymerization in fluidized-bed catalytic reactors. Chinese Journal of Chemical Engineering, 16(1): 84-89

[3] Ibrehem, A.S., Hussain, M.A., \& Ghasem, M.N. (2009). Modified mathematical model for gas phase olefin polymerization in fluidized-bed catalytic reactor. Chemical Engineering Journal , 149: 353-362

[4] Hatzantonis, H., Yiannoulakis, H., Yiagopoulos, A., \& Kiparissides, C. (2000).
Recent developments in modeling gas-phase catalyst olefin polymerization fluidized bedreactor: the effect of bubble size variation on the reactor's performance. Chemical Engineering Science, 5: 3237-3259

[5] McAuley, K.B., MacGregor, J.F., \& Hamielec, A.E. (1990). A kinetic model for industrial gasphase ethylene copolymerization. American Institute of Chemical Engineering Journal, 36: 837-850

[6] Peacock, A.J. (2000). Handbook of Polyethylene. Marcel \& Dekker, New York.

[7] Jamjah, R., Zohuri, G.H., Nekomanesh, M., Javaheri, M., Ahmadjo, S., \& Farhadi, A. (2008). Synthesizing UHMWPE by a ZieglerNatta catalyst based on $\mathrm{MgCl}_{2}$ (ethoxide ty p e)/ $\mathrm{TiCl}_{4} / \mathrm{t} \mathrm{ri}$ - is ob u tyla lu minum, Macromolecular Symposium, 274: 48-153

[8] Dasilva, J.C., Defigueiredo, G.M.O. (2002). Spherical catalyst, process for preparing a spherical polyethylene of ultra-high molecular weight, US Patent. 6,384,163.

[9] Shiraishi, T., Uchida, W., Matsuura, K. (1990). Process for preparing ultra-high molecular weight polyethylene, US Patent. 4,962,164.

[10] Pater, J.T.M., Weickert, G., van Swaaij, W.P.M. (2003). Polymerization of liquid propylene with a fourthgeneration ZieglerNatta catalyst: influence of temperature, hydrogen, monomer concentration, and prepolymerization method on powder morphology. Journal Applied Polymer Science, 87: 1421-1435

[11] Jamjah, R., Zohuri, G.H., Vaezi, J., Ahmadjo, S., Nekoomanesh, M., \& Pouryari, M. (2006). Morphological study of spherical $\mathrm{MgCl}_{2} \cdot \mathrm{nEtOH}$ supported $\mathrm{TiCl}_{4}$ ene. Journal Applied Polymer Science, 101: 3829-3834

[12] Zohuri, G.H., Jamjah, R., Ahmadjo, S. (2006). Comparative study of propylene polymerization using monosupported and bisupported titanium-based Ziegler-Natta catalysts. Journal Applied Polymer Science, 100: $2220-2226$

[13] Moore, E.P. (2005). Polypropylene Handbook, Hanser, Munich.

[14] Galli, P., Vecellio, G. (2004). Polyolefins: the most promising large-volume materials for the 21st century, Journal Applied Polymer Science Part A: Polymer Chemical, 42: 396-415

[15] Barbe, P.C., Cecchin, G., Noristi, L. (1986). The catalytic system Ti-complex/ $\mathrm{MgCl}_{2}$, Advance Polymer Science, 81: 1-81. 
[16] Silva, F.M., Broyer, J.P., Novat, C., Lima, E.L., Pints, J.C,, \& McKenna, T.F. (2005). Investigation of catalyst fragmentation in gas-phase olefin polymerization: a novel short stop reactor. Macromolecular Rapid Communication, 20: 1846-1853

[17] Shaotian, W., Liu, J.C., Make, M.P., Lee, C.C. (2001). Ethylenepolymerization process, US Patent 625,541,5.

[18] Kono, H., Mori, H., Terano, M. (2001). Novel olefin block copolymer, polypropene-blockpoly (methylene-1,3-cyclopentane-copropene), synthesized from propene and 1,5hexadiene by a modified stopped-flow method, Macromolecular Chemical Physics, 202: 1319-1326

[19] Yamahiro, M., Mori, H., Nitta, K.H., \& Terano, M. (1999). Synthesis and basic characteristics of polypropene-blockpoly(ethene-co-propene) by modified stoppedflow polymerization with an $\mathrm{MgCl}_{2}$ supported Ziegler catalyst, Macromolecular Chemical Physics, 200: 134-141
[20] Fukuda, K., Liu, B., Nakatani, H., Nishiyama, I., Yamahiro, M., \& Terano, M. (2003). Significant variation of molecular weight distribution (MWD) of polyethylene induced by different alkyl-Al cocatalysts using a novel surface functionalized $\mathrm{SiO}_{2}$ supported Ziegler-Natta catalyst, Catalyst Communication, 4: 657-662.

[21] Zohuri, G.H., Azimfar, F., Jamjah, R., Ahmadjo, S. (2003). Polymerization of propylene using the high-activity ZieglerNatta catalyst system $\mathrm{SiO}_{2} / \mathrm{MgCl}_{2}$ (ethoxidetype)/TiCl4/Dinbutylphthalate/triet hylaluminum/dimethoxy methyl cyclohexyl silane. Journal Applied Polymer Science, 89: 1177-1181

[22] Zohuri, G.H., Ahmadjo, S., Jamjah, R., Nekoomanesh, M. (2001). Structural study of mono- and bi-supported Ziegler-Natta catalysts of $\mathrm{MgCl}_{2} / \mathrm{SiO}_{2} / \mathrm{TiCl}_{4} /$ donor systems. Iran Polymer Journal, 10: 149-155 